\title{
RNAi: Gene Silencing Approach in C. elegans and H. sapiens
}

\author{
Gomase V.S. ${ }^{*}$ and More R.P. \\ *School of Technology, SRTM University, Sub-Center, Latur, India, gomase.viren@gmail.com \\ Department of Biotechnology, Shivchhatrapati College, Aurangabad, MS, India
}

\begin{abstract}
RNAs (Short interfering RNAs) and miRNAs (microRNAs), which are mediate silencing via distinct mechanisms $C$. elegans and Homo sapiens. The breaking of a doublestranded RNA (dsRNA) matching a specific gene sequence into short pieces called short interfering RNA, which trigger the degradation of mRNA that matches its sequence. In this review, we discussed the RNA interference with its principle and application in recent biological research areas.
\end{abstract}

\section{Principal}

The phenomenon of RNAi, originally described in the nematode worm $C$. elegans by Fire and colleagues in 1998, has been recognized as a general mechanism in many organisms. Basically, RNAi is induced within the cytoplasm when long, double-stranded RNA (dsRNA) is recognized by Dicer, a multidomain RNase III enzyme. Dicer processes dsRNA into short (21-25 nucleotides) duplexes that are termed siRNAs. Like products of other RNase III enzymes, siRNA duplexes contain $5^{\prime}$ phosphate and $3^{\prime}$ hydroxyl termini, and two single-stranded nucleotide overhangs on their 3'ends. These structural features are important for the entry of siRNAs into the RNAi pathway because blunt-ended siRNAs or those that lack a 5 'phosphate group are ineffective in triggering gene silencing [41]. It is processed by the RNase-III enzyme Dicer into 21-28nucleotide double-stranded siRNA duplexes. The RISC associated enzyme Slicer (Argonaute-2) degrades the target mRNA.

\section{Introduction \\ MicroRNAs}

MicroRNAs are a family of small, non-coding RNAs that regulate gene expression in a sequencespecific manner. The two founding members of the microRNAs family were originally identified in C. elegans as genes that were required for the timed regulation of developmental events [26]. Micro-RNAs have been found to be abundant and phylogenetically extensive in plants, flies, worms, and humans. In $D$. melanogaster, $C$. elegans, plants, and humans, more than 600 microRNAs have been identified [22, 23, 24, and 27]. Bioinformatics analyses of the complete genome sequences have been extremely useful for identification studies [33]. The first step in computational identification of microRNAs from genome sequences is identification of sequences forming hairpin loops (stem-loop sequences). For this purpose, software such as srnloop [15] and RNA fold [25] is used. These are Blast-like software packages, which identify short complementary sequences within a specified distance on the genome [33]. miRNAs are misexpressed in human cancers where they can exert their effect as oncogenes or tumor suppressors. Here, we review the potential for using miRNAs as biomarkers for diagnosis, prognosis and cancer therapies [35].

\section{siRNA (short interfering RNA)}

Gene silencing mechanism that can be mediated either by small interfering RNAs (siRNAs) of about 21 nt with two-nucleotide 3' overhang, or by stably expressed short hairpin RNAs (shRNAs), which are processed by Dicer into siRNAs [7]. The RNA species that binds mRNA in the RNA-induced silencing complex (RISC) then mRNA/siRNA interaction activates the nuclease activity of RISC, leading to degradation of the mRNA. Small interfering RNA (siRNA) is one of the latest additions to the repertoire of sequence-specific gene-silencing agents [46]. The degradation of specific endogenous mRNAs is now recognized as a mechanism that is widely employed by eukaryotic cells to inhibit protein production at a posttranscriptional level [34]. SiRNAs with 2-3 nt overhangs on their 3 ' ends were more efficient in reducing the amount of target mRNA than siRNAs with blunt ends [13]. The following approach recommends for designing a siRNA [34]. 1. Beginning with the AUG start codon of the target gene transcript, scan downstream for $A A$ dinucleotide sequences; each AA and the 3 adjacent 19 nucleotides are potential siRNA targets. 
2. Compare the sequences of the potential target sequences to sequences in the speciesappropriate genome database (www.ncbi.nlm.nih.gov/BLAST/) and eliminate from consideration any target sequences that are homologous to other coding sequences.

3. Select 3-4 target sequences along the length of the gene for production of siRNAs. Of course it is important for all siRNA experiments to include negative control siRNAs with the same nucleotide composition but a scrambled sequence. Further analyses revealed that application of an algorithm incorporating all eight criteria significantly improves potent siRNA selection [38]. The siRNA construct builder is used to create a siRNA hairpin construct as a vector insert or a cassette insert. These programs provide a comprehensive utility set to address the specific bioinformatics need of DNA-based siRNA design [28]. There are some developer offers tools to design siRNA. Some of them listed below [36].

Table No. 1- List of Developer \& its URL address.

\begin{tabular}{|l|l|}
\hline Developer & Web Site ID \\
\hline Ambion & www.ambion.com/techlib/misc/siRNA_finder.html \\
\hline Dharmacon & http://design.dharmacon.com// \\
\hline Clontech & http://bioinfo2.clontech.com/rnaidesigner/ \\
\hline Deqor & http://cluster-1.mpi-cbg.de/Deqor/deqor.html \\
\hline dsCheck & http://alps3.gi.k.u-tokyo.ac.jp/ dscheck/main/index2.php \\
\hline EMBOSS & http://inn.weizmann.ac.il/EMBOSS \\
\hline GenScript & www.genscript.com/ssl-bin/app/rnai \\
\hline Hannon Lab & http://katahdin.cshl.org:9331/siRNA/ \\
\hline IDTDNA & http://biotools.idtdna.com/rnai// \\
\hline Interagon & www.interagon.com/demo/ \\
\hline Invitrogen & https://rnaidesigner.invitrogen.com/sirna/ \\
\hline Jack Lin & www.sinc.sunysb.edu/Stu/shilin/rnai.html \\
\hline OptiRNAi & http://bioit.dbi.udel.edu/rnai// \\
\hline Promeg & www.promega.com/siRNADesigner/program/ \\
\hline Qiagen & www.qiagen.com/Products/GeneSilencing/ \\
\hline SFold & http://sfold.wadsworth.org/sirna.pl \\
\hline SiDE 20 & http://side.bioinfo.ochoa.fib.es/ \\
\hline siDirect & http://design.rnai.jp/sidirect// \\
\hline siRNA wizard & http://www.sirnawizard.com/design_advanced.php \\
\hline siSearch & http://sonnhammer.cgb.ki.se/siSearch/siSearch_1.6.html \\
\hline TROD & http://websoft2.unige.ch/sciences/biologie/bicel/RNAi \\
\hline
\end{tabular}

Table 2- The Advantages and Disadvantages of siRNA are given below [4].

\begin{tabular}{|l|l|}
\hline Advantages & Disadvantages \\
\hline Consistent quality of reagents & Finite resource. \\
\hline Ease of use and readily transfectable & Target cells need to be transfectable. \\
\hline $\begin{array}{l}\text { Chemical modification of siRNA can limit } \\
\text { off-target effects. }\end{array}$ & Relatively short period of silencing. \\
\hline
\end{tabular}

RNA Interference in C. elegans

RNA interference (RNAi) was originally discovered in Caenorhabditis elegans as a mechanism of posttranscriptional gene silencing induced by exogenous dsRNA. It is now known that RNAirelated mechanisms play roles in inhibition of target mRNA translation, mRNA degradation, or repression of transcription. RNAi has been cultivated as a means to manipulate gene expression experimentally and to probe gene function on a whole-genome scale [16]. Recent studies showed that surprisingly, both RNAi and miRNA pathway genes affect RNAi-induced transcriptional gene silencing (RNAi-TGS) in the soma of $C$. elegans [1]. RNAi-mediated gene silencing can also function through repressive chromatin modifications in some organisms [2]. A majority of human cancers have a mutation inactivating the retinoblastoma $(\mathrm{Rb})$ pathway. Three Rb-related genes International Journal of Molecular Biology, ISSN: 0976-0482 \& E-ISSN: 0976-0490 
are expressed in vertebrate cells, but only one member of this family exists in $C$. elegans. In both types of organisms Rb-related activities are thought to control cell proliferation and development primarily by suppressing transcription at promoters near their binding sites [1]. RNAi among nematodes, efficiency of RNA interference is far from general, however, even in nematodes. Until recently, the use of bacteria expressing dsRNAs in nematodes was restricted to $C$. elegans. Even within the Caenorhabditis genus [30]. The use of RNA interference to inhibit the function of $86 \%$ of the 19,427 predicted genes of C. elegans [37]. Micro array expression profiling of RNAidefective mutant worms further suggests diverse endogenous RNAi pathways for silencing different sets of genes. The lin- 4 and let- 7 antisense RNAs are temporal regulators that control the timing of developmental events in C. elegans by inhibiting translation of target mRNAs [38, 39].

\section{RNA Interference in Homo sapiens}

RNAi is an evolutionarily conserved mechanism involved in the post-transcriptional regulation of gene expression in many eukaryotes. It was initially recognized as an anti-viral mechanism that protected organisms from RNA viruses or the random integration of transposable elements [3]. The ability of RNAi to inhibit the replication or cellular uptake of viruses and other infectious agents has been clearly demonstrated in cell culture studies and, therefore, holds promise for the treatment of human patients [34]. A MBD1 knock-down BxPC-3 pancreatic cancer cell line using a stable RNAi method. The comparative proteomic approach is a useful strategy of proteomics to analyze and compare the differentially expressed proteins and identify novel target molecules in unknown pathways [6]. RNAi screen to identify novel determinants of response to the commonly used breast cancer therapy, tamoxifen.20 Breast tumour cells were transfected with siRNA and then divided into tamoxifen-treated and control groups [4]. Recent transcriptome analyses have shown that most of the eukaryotic genome is transcribed, and the genomes of all cellular life forms, in addition to protein-coding genes, contain varying numbers of non-protein-coding RNA [42]. The effects of RNAi in reducing the target gene expression in human myeloid leukemia cell lines [9]. The main biological role of such silencing is usually to protect from invasive nucleic acids including viruses and retrotransposons, and to maintain the chromosomal stability [17]. Human let-7 is a component of a previously identified, miRNA-containing ribonucleoprotein particle, which we show is an RNAi enzyme complex [18].

\section{Methods}

Initially Dicer-RNase III-like enzyme cleaves dsRNA into siRNA. siRNAs are then incorporated into RNA-Induced Silencing Complexes (RISCs) and unwinds. The RISC probes subsequently target RNAmediated cleavage of complementary mRNA inducing gene specific silencing [19].

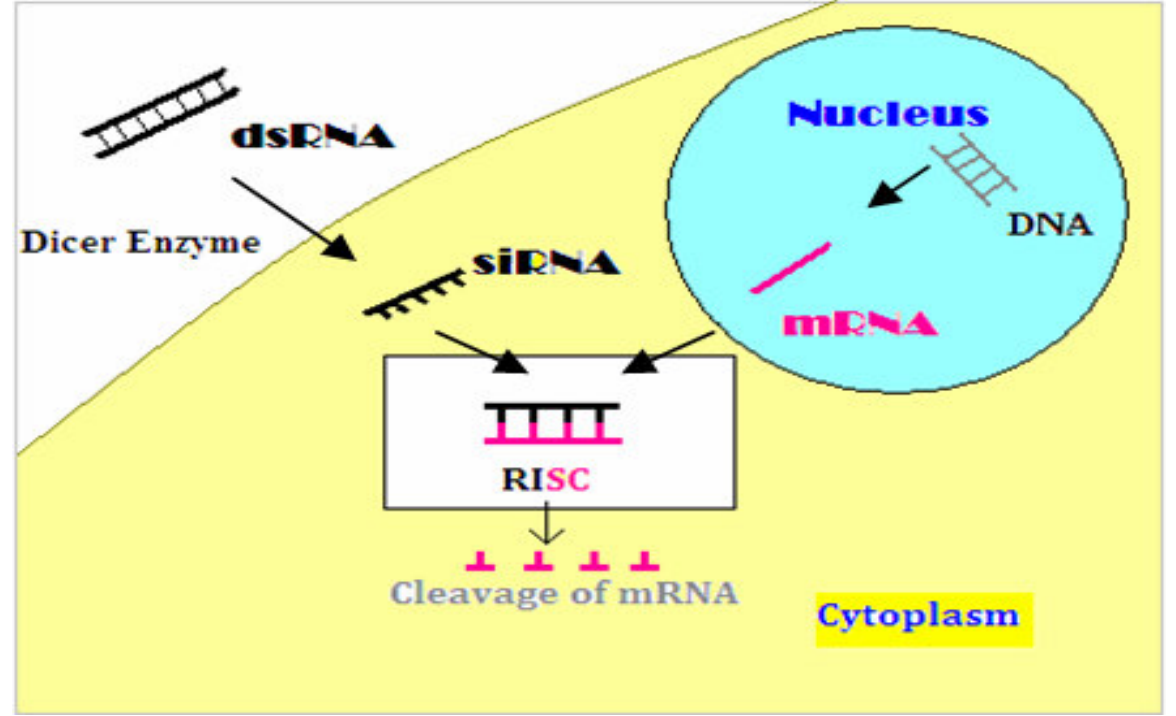

Fig. 1- RNAi is a process in which siRNA molecules activate a cellular process that results RNAInduced Silencing Complex (RISC) then cleavage of a specific mRNA. 


\section{Applications of RNAi- to improve drug candidate}

Many different types of chemical modification have been developed to improve the in vivo properties of nucleic acids. Each modification offers a different potential for tailoring the properties of siRNAs such as Increased thermal stability, Increased stability to digestion by nucleases, Improved biodistribution, Improved biodistribution, Targeting specific tissue, Specificity for target mRNA [11]. RNAi also having potential for treating diseases like oral diseases. The advantages of efficient, economical knockdown offered by RNAi [36] Many RNAi screens have been carried out with the aim of exploring the mechanism of resistance to chemotherapeutic drugs [4]. RNAi is lauded as a powerful approach to gene control, with the promise of revolutionizing basic research and providing treatments for intractable conditions such as cancer and neurodegenerative disease [21].

\section{In clinical trials}

The regulatory complexities of miRNAs should also be taken into consideration when either ablation or restoration of miRNA function is being considered in a therapeutic setting. A single miRNA can regulate the levels of hundreds of proteins, raising cautionary flags about the consequences of down regulating or ectopically expressing even a single miRNA species [10].

\section{Cancer RNAi techniques}

C. elegans can help gathering insights on cellular mechanisms leading to tumour formation. Two microRNAs, miR-84 and miR-61, control the expression of the oncogene orthologues Ras and Vav indicating their capacity to act as tumour suppressors. These observations demonstrate that uncovering the function of microRNAs is important to increase our understanding of cancer [12]. The use of RNA interference libraries can be used for high-throughput screening on a genebygene basis to find those that underlie the characteristics of malignant transformation [20]. RNAi cancer technology include the inhibition of over expressed oncogenes, blocking cell division by interfering with cyclin $\mathrm{E}$ and related genes or promoting apoptosis by suppressing antiapoptotic genes. RNAi against multidrug resistance genes or chemoresistance targets may also provide useful cancer treatments [31]. Knockdown of AURKB and EGFR individually resulted in inhibition of prostate tumor growth in athymic nude mice and their combined knockdown resulted in tumor regression [29].

\section{Therapeutic applications}

RNAi likely developed as an endogenous host defense mechanism directed against viral infections. Evidence for this has been seen in both plants and animals, such as $C$. elegans and Drosophila. These strategies could be used for therapeutic purposes [5]. The goals for RNAi approaches for cancer therapy are therefore to knock out the expression of a cell cycle gene and/or an anti-apoptotic gene in the cancer cells thereby stopping tumor growth and killing the cancer cells [34]. The clinical potential of RNAi is powerful tool in various diseases including viral infections, cancer and neurodegenerative disorders [44]. The first siRNA, bevasiranib (Opko Health; formerly named Cand5 from Acuity Pharmaceuticals), has recently entered a key Phase III trial for the treatment of wet age-related macular degeneration (AMD) [36].

\section{Immune system studies}

The innate immune system was originally considered an evolutionarily primitive preliminary response to infection. However, as this system was further explored, it became clear that the innate immune system is composed of highly sophisticated defense mechanisms and that it is crucial in initiating the adaptive immune system [8]. RNAi technologies have progressed in such a rapid rate that already applications have been filed with the FDA for approval of clinical trials with siRNA molecules [44]. The identification of components of the RNAi machinery as targets of the anti-Su autoantibody system provides another example of an autoimmune response directed at a macromolecular complex that plays a key role in the posttranscriptional regulation of gene expression [14]. Autoimmunity has the potential to advance in understanding of autoimmune responses to components of the RNAi pathway. Interestingly, pristane treatment of mice has been demonstrated to activate endogenous retroviruses [3]. RNAi technology has also recently 
yielded significant insight into the innate and adaptive immune systems by helping to elucidate numerous mechanisms that regulate the development, activation and function of cells that mediate immunity [8]. To identify sequence-based modifications allowing for the design of bifunctional siRNAs with both proinflammatory and specific silencing activities, and with potentially increased therapeutic benefits and the introduction of a micro-RNA (miRNA)-like nonpairing uridine-bulge in the passenger strand robustly increased immunostimulatory activity on human immune cells [32].

\section{In the Pathology}

RNAi screens represent more of a research tool rather than an application that could be used in the diagnostic laboratory [4]. Numerous studies have documented the implications of miRNAs in nearly every pathological process of the cardiovascular system, including cardiac arrhythmia, cardiac hypertrophy, heart failure, cardiac fibrosis, cardiac ischemia and vascular atherosclerosis [47].

\section{The Future Prospective of RNAi in Biological Research}

The molecular role of RNAi and in the development of methods for the use of RNAi technology for selective gene silencing in eukaryotic species, due to the availability of whole genome sequences of eukaryotic species.

\section{Conclusion}

RNA interference plays an important role in many cellular processes, including viral defense, chromatin remodeling, genome rearrangement and gene expression. These studies have focused on the functions of critical genes, immune cell development and differentiation, and initiator and effectors mechanisms of immune responses and in different research areas of biology. RNA interference has also contributed to the understanding of expression of genes thought out the eukaryotic genome. RNAi offers a quick and easy way to determine its function RNAi may furthermore be used to therapeutic agent to find drug candidates specifically in drug discovery. In this review article, we examined the current progress of RNAi in C. elegans and Homo sapiens species. Also, Gene silencing using RNAi additionally demonstrates it can helpful in research areas like Drug discovery, Immunology, pathology and its related areas. As a research tool, RNAi has the advantage of knocking down gene expression without altering genomic DNA structure and function.

\section{References}

[1] Alla Grishok and Phillip A. Sharp. Negative regulation of nuclear divisions in Caenorhabditis elegans by retinoblastoma and RNA interference-related genes: PNAS (2005) 102, 48, 17360-17365.

[2] Alla Grishok, Sebastian Hoersch, and Phillip A. Sharp, 'RNA interference and retinoblastoma-related genes are required for repression of endogenous siRNA targets in Caenorhabditis elegans: PNAS 2008 105, 51, 20386-20391.

[3] Andrew Jakymiw, Keigo Ikeda, Marvin J Fritzler. Autoimmune targeting of key components of RNA interference: Arthritis Research \& Therapy (2006) 8, R87.

[4] C. J. Lord, S. A. Martin, A. Ashworth. RNA interference screening demystified: J Clin Pathol (2009) 62, 195-200.

[5] Carol A. Sledz and Bryan R. G. Williams. RNA interference in biology and disease: Blood (2005) 106, 3, 787-794.

[6] Chen Liu, Yaohui Chen, Xianjun Yu. Proteomic analysis of differential proteins in pancreatic carcinomas: Effects of MBD1 knock-down by stable RNA interference: BMC Cancer (2008), 8,121.

[7] Chi Yu Chan, C Steven Carmack. A structural interpretation of the effect of GC-content on efficiency of RNA interference', BMC Bioinformatics (2009), 10, 1, S33.

[8] Chih-Ping Mao, Yen-Yu Lin, Chien-Fu Hung. Immunological research using RNA interference technology: Immunology (2007) 121, 295-307. 
[9] Daniel P Cioca, Yuji Aoki and Kendo Kiyosawa. RNA interference is a functional pathway with therapeutic potential in human myeloid leukemia cell lines: Cancer Gene Therapy (2003) 10, 125-133.

[10] Daniela Castanotto and John J. Rossi. The promises and pitfalls of RNA-interference-based therapeutics: Nature (2009) 457, 7228, 426-433.

[11] David R. Corey. Chemical modification: the key to clinical application of RNA interference?: J. Clin. Invest. (2007) 117, 3615-3622.

[12] G Jannot \& M J Simard. Tumour-related microRNAs functions in Caenorhabditis elegans: Oncogene (2006) 25, 6197-6201.

[13] George L. Sen and Helen M. Blau. A brief history of RNAi: the silence of the genes: The FASEB Journal (2006) 20, 1293-1299.

[14] Ger JM Pruijn. The RNA interference pathway: a new target for autoimmunity: Arthritis Res Ther. (2006) 8, 4, 110. PMCID: PMC1779402

[15] Grad, Y., et al, Computational and experimental identification of C. elegans micro-RNAs: Mol. Cell (2003) 11, 1253-1263.

[16] Gregory J. Hannon. Review article RNA interference: Nature (2002) 418, 244-251.

[17] H Akashi and K Taira. RNAi and epigenetics: Pol IV is a matchmaker of small RNAs meeting with chromatin: Heredity (2007) 98, 125-127.

[18] Hutvagner, G., and P. D. Zamore. A micro-RNA in a multiple-turnover RNAi enzyme complex: Science (2002) 297, 2056-2060.

[19] Joanne Lomas-Neira, Chun-Shiang Chung and Alfred Ayala. RNA Interference as A Potential Therapeutic Treatment for Inflammation Associated Lung Injury: Int J Clin Exp Med (2008) 1, 154-160.

[20] Julian Downward. RNA interference-based functional genomics in cancer research - an introduction: Oncogene (2004) 23, 8334-8335.

[21] Julie Clayton. RNA interference: The silent treatment: Nature (2004) 431, 599-605.

[22] Lagos-Quintana, R. Rauhut, W. Lendeckel, and T. Tuschl. Identification of novel genes coding for small expressed RNAs: Science (2001) 294, 853-858.

[23] Lau, N. C., L. P. Lim, E. G. Weinstein, and D. P. Bartel. An abundant class of tiny RNAs with probable regulatory roles in Caenorhabditis elegans: Science (2001) 294,858-862.

[24] Lee, R. C., and V. Ambros. An extensive class of small RNAs in Caenorhabditis elegans: Science (2001) 294, 62-864.

[25] Lim, L. P., N. C. Lau, E. C. Weinstein, A. Abdelhakim, S. Yekta, M. W. Rhoades, C. B. Burge, and D. P. Bartel. The micro-RNAs of C. elegans: Genes Dev. (2003) 17, 9911008.

[26] Lin He \& Gregory J. Hannon. MicroRNAs: small RNAs with a big role in gene regulation: Nature Reviews Genetics (2004) 5, 522-531.

[27] Llave, C., K. D. Kasschau, and J. C. Carrington. Virus encoded suppressor of posttranscriptional gene silencing targets a maintenance step in silencing pathway: Proc. Natl. Acad. Sci. USA (2000) 97, 13401-13406.

[28] Luquan Wang and Forest Y. Mu. A Web-based design center for vector-based siRNA and siRNA cassette: Bioinformatics (2004) 20, 11, 1818-1820.

[29] M K Addepalli, et al. RNAi-mediated knockdown of AURKB and EGFR shows enhanced therapeutic efficacy in prostate tumor regression: Gene Therapy (2009) doi:10.1038/gt.2009.155

[30] Marie-Anne Félix. RNA interference in nematodes and the chance that favored Sydney Brenner: Journal of Biology (2008) 7, 34.

[31] Marta Izquierdo. Short interfering RNAs as a tool for cancer gene therapy: Cancer Gene Therapy (2005) 12, 217-227.

[32] Michael P Gantier, et al. Rational Design of Immunostimulatory siRNAs', Molecular Therapy (2010)

[33] Neema Agrawal, et al. RNA Interference: Biology, Mechanism, and Applications: MMBR (2003) 67, 4, 657-685.

[34] OLLIVIER MILHAVET, DEVIN, MARK. RNA Interference in Biology and Medicine: Pharmacol Rev (2003) 55, 629-648. 
[35] P Trang, J B Weidhaas \& F J Slack. MicroRNAs as potential cancer therapeutics: Oncogene (2008) 27, S52-S57.

[36] P. N. Pushparaj, et al. siRNA, miRNA, and shRNA: in vivo Applications: J DENT RES (2008) 87, 11, 992-1003.

[37] Ravi S. Kamath, Andrew G. Fraser. Systematic functional analysis of the Caenorhabditis elegans genome using RNAi: Nature (2003) 421, 231-237.

[38] Reynolds A, Leake D, Boese Q, Scaringe S, Marshall WS, Khvorova A. Rational siRNA design for RNA interference: Nat Biotechnol. (2004) 22, 3, 326-30.

[39] Rosalind C. et al. Interacting endogenous and exogenous RNAi: RNA (2006) 12, 4, 589597.

[40] Rosalind C. Lee, Victor Ambros. An Extensive Class of Small RNAs in Caenorhabditis elegans: Science (2001) 294, 5543, $862-864$.

[41] Sascha Rutz and Alexander Scheffold. Towards in vivo application of RNA interference new toys, old problems: Arthritis Res Ther (2004) 6, 78-85.

[42] Svetlana A. Shabalina and Eugene V. Koonin. Origins and evolution of eukaryotic RNA interference: Trends Ecol Evol. (2008) 23, 10, 578-587.

[43] Thoru Pederson. RNA Interference and mRNA Silencing: How Far Will They Reach?: Molecular Biology of the Cell (2004) 15, 2, 407-410.

[44] Tom C Karagiannis, Assam El-Osta. RNA interference and potential therapeutic applications of short interfering RNAs: Cancer Gene Therapy (2005) 12, 787-795.

[45] Wallace F. Marshall. Modeling Recursive RNA Interference: PLoS Computational Biology (2008) 4, 9, e1000183.

[46] Yair Dorsett \& Thomas Tuschl. siRNAs: applications in functional genomics and potential as therapeutics: Nature Reviews Drug Discovery (2004) 3, 318-329.

[47] Zhen-wei PAN, Yan-jie LU, Bao-feng YANG. MicroRNAs: a novel class of potential therapeutic targets for cardiovascular diseases: Acta Pharmacologica Sinica (2010) $31,1-9$. 